\title{
Application of a novel liquid biopsy in patients with hepatocellular carcinoma undergoing liver transplantation
}

\author{
FENG XUE $^{1 *}$, SHAOJUN SHI $^{1 *}$, ZHENZHEN ZHANG $^{2}$, CHENG XU $^{2}$, JIANXIN ZHENG $^{1}$, \\ TIAN QIN $^{1}$, ZHIYU QIAN $^{1}$, XIAOYU ZHAO $^{2}$, YING TONG $^{1}$, LEI XIA $^{1}$ and QIANG XIA ${ }^{1}$ \\ ${ }^{1}$ Department of Liver Surgery and Liver Transplantation Center, Renji Hospital, School of Medicine, \\ Shanghai Jiaotong University, Shanghai 200127; ${ }^{2}$ Zhangjiang Center for Translational Medicine, \\ Biotecan Medical Diagnostics Co., Ltd, Shanghai 200123, P.R. China
}

Received June 14, 2017; Accepted December 4, 2017

DOI: $10.3892 / \mathrm{ol} .2018 .8019$

\begin{abstract}
Circulating tumor cells (CTCs) serves a primary function in metastasis and recurrence of hepatocellular carcinoma (HCC). In the present study, in order to evaluate the analytical performance and clinical value of the liquid biopsy-based platform, a novel integrated subtraction enrichment and immunostaining-fluorescence in situ hybridization $\left(\mathrm{iFISH}^{\circledR}\right)$ platform was applied to analyze CTCs in patients with HCC undergoing liver transplantation (LT). In total, 30 patients with $\mathrm{HCC}$ undergoing LT and 10 healthy volunteers were enrolled. CTCs in peripheral blood that were obtained from each patient prior to LT and 3 months thereafter were detected using the iFISH ${ }^{\circledast}$ platform, and CellSearch ${ }^{\circledR}$ system was performed for each subject for comparison. Using iFISH ${ }^{\circledR}$ and CellSearch ${ }^{\circledast}$, the percentage of CTCs in patients with pre-operative HCC were $70.00 \%$ and $26.67 \%$, respectively. CTCs counted using iFISH ${ }^{\circledR}$ (iFISH-CTCs) were increased compared with CellSearch ${ }^{\circledR}($ Cellsearch-CTCs) $(\mathrm{P}<0.01)$. A
\end{abstract}

Correspondence to: Dr Qiang Xia, Department of Liver Surgery and Liver Transplantation Center, Renji Hospital, School of Medicine, Shanghai Jiaotong University, 1630 Dongfang Road, Shanghai 200127, P.R. China

E-mail: xiaqiang@shsmu.edu.cn

${ }^{*}$ Contributed equally

Abbreviations: AFP, $\alpha$-fetoprotein; CTC, circulating tumor cells; Cellsearch-CTCs, CTCs counted by CellSearch ${ }^{\circledR}$; CEP8; Chromosome 8; CKs, cytokeratins; CT, contrast enhanced computed tomography; DAPI, 4',6-diamidino-2-phenylindole; EMT, epithelial-mesenchymal transition; EpCAM, epithelial cell adhesion marker; HCC, hepatocellular carcinoma; iFISH, immunostaining-fluorescence in situ hybridization; iFISH-CTCs, CTCs counted by iFISH; LT, liver transplantation; MRI, magnetic resonance imaging; RBCs, red blood cells; RFS, recurrence-free survival; ROC, receiver operating characteristic; SEN, sensitivity; WBCs, white blood cells

Key words: circulating tumor cell, hepatocellular carcinoma, liver transplantation, subtraction enrichment, liquid biopsy, recurrence significant decrease in iFISH-CTCs was observed 3 months following LT $(3.04 \pm 0.93 / 7.5$ to $1.0 \pm 0.53 / 7.5 \mathrm{ml}, \mathrm{P}<0.05)$. Furthermore, patients with lower preoperative iFISH-CTCs level $(<5 / 7.5 \mathrm{ml})$ had markedly increased recurrence-free survival compared with iFISH-CTCs $(>5 / 7.5 \mathrm{ml}, 15$ vs. 5.5 months; $\mathrm{P}<0.01$. FISH $^{\circledR}$ platform exhibits an increased analytical sensitivity, and may be used as a dynamic monitoring tool for CTCs, and CTCs may be a good prognostic indicator for patients with HCC undergoing LT.

\section{Introduction}

Hepatocellular carcinoma (HCC) is one of the most prevalent malignancies worldwide and was the second leading cause of cancer-associated mortality in 2011 (1). The incidence rate of HCC is the highest in east and Southeast Asia, as well as in Middle and Western Africa; however, it is also becoming more prevalent in the United States and Europe (1). Despite liver transplantation (LT) remaining one of the most effective radical treatments, a significant proportion of patients still suffer from unfavorable outcomes due to a high recurrence rate ( $15-20 \%)(2,3)$. Ultrasonography has been widely used in HCC screening and monitoring; however, interpretation of test results is subjective and depends upon the skill and experience of investigators and therefore this limits its clinical application. Likewise, radiological examinations including contrast enhanced computed tomography (CT) or magnetic resonance imaging (MRI) are sometimes not able to detect small tumors and inconspicuous metastasis lesions due to low sensitivity, leading to delayed treatment. $\alpha$-fetoprotein (AFP) is a widely used serum marker in the clinical diagnosis and surveillence of HCC. However, it has been previously reported that pregnancy, benign liver disease and certain gastrointestinal tumors may contribute to false-positive results (4). Therefore, the importance of developing an accurate diagnostic marker or monitoring tool for patients with HCC should be emphasized.

Circulating tumor cells (CTCs) are cells that have shed into the vasculature from a primary or metastatic solid tumor and are carried around the body via blood circulation. Previous studies have demonstrated that CTCs serve a key function in metastasis and recurrence in HCC $(5,6)$. Due to the complete 
removal of tumor lesion(s) and implantation of tumor-free grafts through LT, the source of short-term recurrence and metastasis must be residual extrahepatic CTCs. Therefore, investigation of CTCs in peripheral blood, also termed liquid biopsy, is a promising strategy for monitoring patients with HCC undergoing LT.

CellSearch ${ }^{\circledR}$ system is a traditional device for CTCs detection (7). High-level CTCs detected by CellSearch ${ }^{\circledR}$ system have been implicated in increased recurrence risk in HCC patients after liver resection $(8,9)$. However, previous evidence demonstrated that epithelial-mesenchymal transition (EMT) process may contribute to the low expression of epithelial cell adhesion marker (EpCAM) and cytokeratins (CKs), and thus reduce the detection sensitivity (10). The immunostaining-fluorescence in situ hybridization ${ }^{\circledR}\left(\mathrm{iFISH}^{\circledR}\right)$ platform exhibits good sensitivity in breast (7), gastric (8), lung (9) and pancreatic cancer (10), and is therefore highly recommended. However, the size of tumor cells and EpCAM expression varies. iFISH can eliminate red blood cells (RBCs) and deplete white blood cells (WBCs) using anti-CD45 antibodies, then in situ phenotypic and karyotypic identification is performed using centromere probe 8 (CEP8), which effectively improves CTCs detection sensitivity via subtractive enrichment (11). In the present study, $\mathrm{iFISH}^{\circledR}$ was utilized to separate and characterize CTCs in patients with HCC undergoing LT. The present study aims to evaluate the analytical performance of iFISH ${ }^{\circledR}$ and examine it's clinical value, as well as compare it to the CellSearch ${ }^{\circledR}$ system. To the best of our knowledge, this is the first time that this platform has been applied to patients with HCC undergoing LT.

\section{Materials and methods}

Patients and sample collection. Between November 2014 and October 2015, peripheral blood samples were collected from 30 HCC patients undergoing LT, and 10 healthy controls in Renji Hospital (Shanghai, China). Blood was obtained 2 days prior to transplantation for patients with HCC (baseline), and a median of 90 days (range, 81-97 days) following LT. At each time point, $7.5 \mathrm{ml}$ peripheral blood was collected from each subject for iFISH ${ }^{\circledR}$ and Cellsearch ${ }^{\circledR}$ analyses. Blood samples were processed within $48 \mathrm{~h}$ of collection. Ethical approval for the recruitment of human subjects was obtained from the Ethics Committee of Shanghai Jiaotong University Affiliated Renji Hospital (Shanghai, China) and was consistent with ethical guidelines provided by the Declaration of Helsinki (1975). Written informed consent was obtained from each patient prior to collecting blood samples. Clinical information was also collected including the AFP level, size and number of tumors, Barcelona Clinic Liver Cancer (BCLC) stage (12), Milan criteria (13), Child-Pugh score and presence of hepatitis B virus, hepatitis C virus, vascular invasion and portal vein tumor thrombus. Patients were followed up every 3 months during the first postoperative year and at least every 3-4 months thereafter. The mean follow-up was 11.2 months (range, 4-20 months). During the follow-up, 3 patients succumbed to intrahepatic recurrence and 3 patients succumbed to extrahepatic metastasis. All patients were monitored prospectively by measuring levels of serum AFP and performing abdomen ultrasonography monthly in the first 3 months and every 6 months in late stage following LT. Chest x-ray was also performed every 6 months following LT. For patients with test results indicating recurrence, CT and/or MRI, and even bone scanning were used to verify whether intrahepatic recurrence and/or extrahepatic metastasis had occurred. A diagnosis of recurrence was based on typical imaging appearances in CT, MRI and/or bone scans, as well as an elevated AFP level $>400 \mathrm{IU} / \mathrm{ml}$.

Subtraction enrichment in iFISH ${ }^{\circledR}$. Subtraction enrichment of CTCs was performed similarly to previous studies $(7,10,11)$. Cytelligen ${ }^{\circledR}$ CTC enrichment kit (Cytelligen Inc., San Diego, CA, USA) was used to enrich CTCs. Briefly, a $7.5 \mathrm{ml}$ blood sample was collected into acid-citrate-dextrose anticoagulant tube, centrifuged at $600 \mathrm{x}$ g for $5 \mathrm{~min}$ at room temperature and the supernatant was discarded. The sample was mixed with $3 \mathrm{ml}$ Separation Matrix (Cytelligen Inc., San Diego, CA, USA), centrifuged at $400 \mathrm{x} \mathrm{g}$ for $5 \mathrm{~min}$ at room temperature and the white buffy coat was collected and then incubated with $150 \mu \mathrm{l}$ immunomagnetic particles conjugated to anti-cluster of differentiation (CD)45 antibody (SEH-001R3; Cytelligen Inc.) for $10 \mathrm{~min}$ at room temperature, separated by a magnetic separator (cat. no. V8151; Promega Corporation, Madison, WI, USA). The bead-free solution was transferred to a centrifuge tube and washed twice with CRC washing buffer (SEH-001R1; Cytelligen Inc.) and, centrifuged at $650 \mathrm{x} \mathrm{g}$ for $5 \mathrm{~min}$ at room temperature. The supernatant was removed, then $100 \mu$ l Cytelligen Fixative (SEH-001R4; Cytelligen Inc.) was added at room temperature for $4 \mathrm{~h}$ and the cell suspension was placed into cover glass, $37^{\circ} \mathrm{C}$ overnight for drying.

Immunofluorescent staining of CTCs in iFISH ${ }^{\circledR}$. CTCs identification was performed using the Cytelligen ${ }^{\circledR}$ CTCs identification kit (Cytelligen Inc.) according to the manufacturers' protocol. Samples were prepared and fixed as aforementioned. Briefly, the cover glass was removed in FR2 buffer (FSH-001R2; Cytelligen Inc.) and dehydrated in 100\% ethanol for $2 \mathrm{~min}$ in room temperature. CEP 8 SpectrumOrange (Vysis, Inc.; Abbott Pharmaceutical Co. Ltd., Lake Bluff, IL, USA) hybridization was performed according to a previously published protocol (7) Slides were then were then incubated with antibody preparation solution-1 (FSH-001R6; Cytelligen Inc.), anti-cytokeratin 18 antibody (HAB-001R1; Cytelligen Inc.) and anti-CD45 antibody (HAB-001R2; Cytelligen Inc.). Finally, mounting medium (FSH-001R7; Cytelligen Inc.) with DAPI was added and observed using a fluorescence microscope (Magnification, x40; Nikon Corporation, Tokyo, Japan). CTCs were defined as cells with features of CK+/CD45-/DAPI+ and hybridization signal for CEP8 $\geq 2$, CK-/CD45-/DAPI+ and hybridization signal for CEP8>2. CK-/CD45+/DAPI+ and hybridization signal for $\mathrm{CEP} 8=2$ was defined as a white blood cell (WBC) and CK-/CD45-/DAPI+ and hybridization signal for $\mathrm{CEP} 8=2$ was defined as an indeterminate cell.

CTCs detected using CellSearch ${ }^{\circledR}$ system. Samples were detected using CellSearch ${ }^{\circledR}$ system according to the manufacturer's protocol (Menarini Silicon Biosystems, Inc., San Diego, CA, USA). Prior to testing, $7.5 \mathrm{ml}$ of peripheral blood was collected (as aforementioned) into the CellSave Preservative 


\section{A}
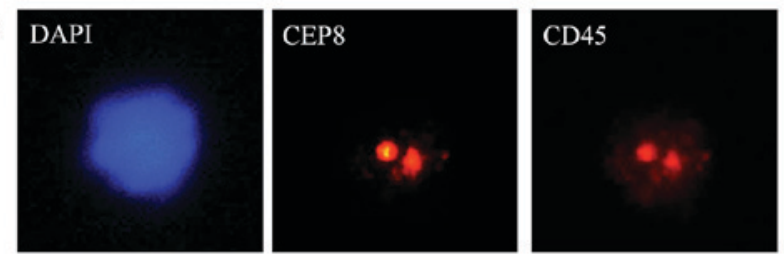

B
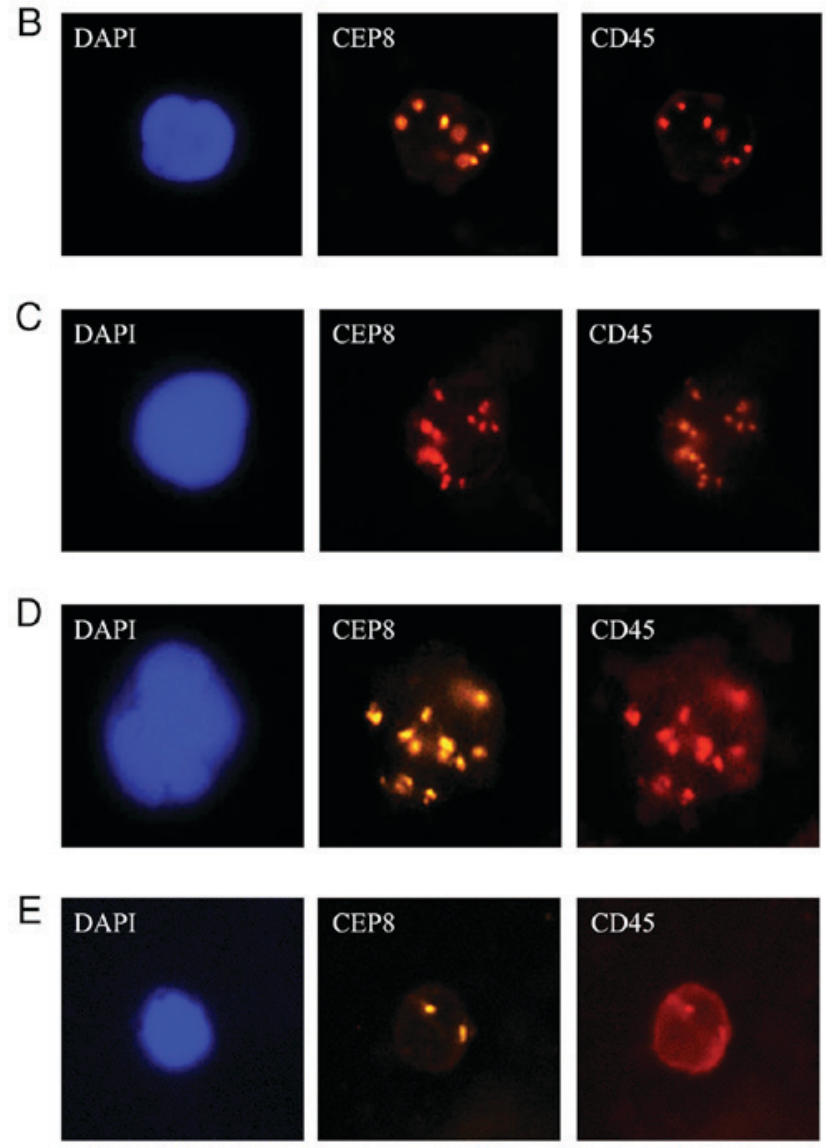
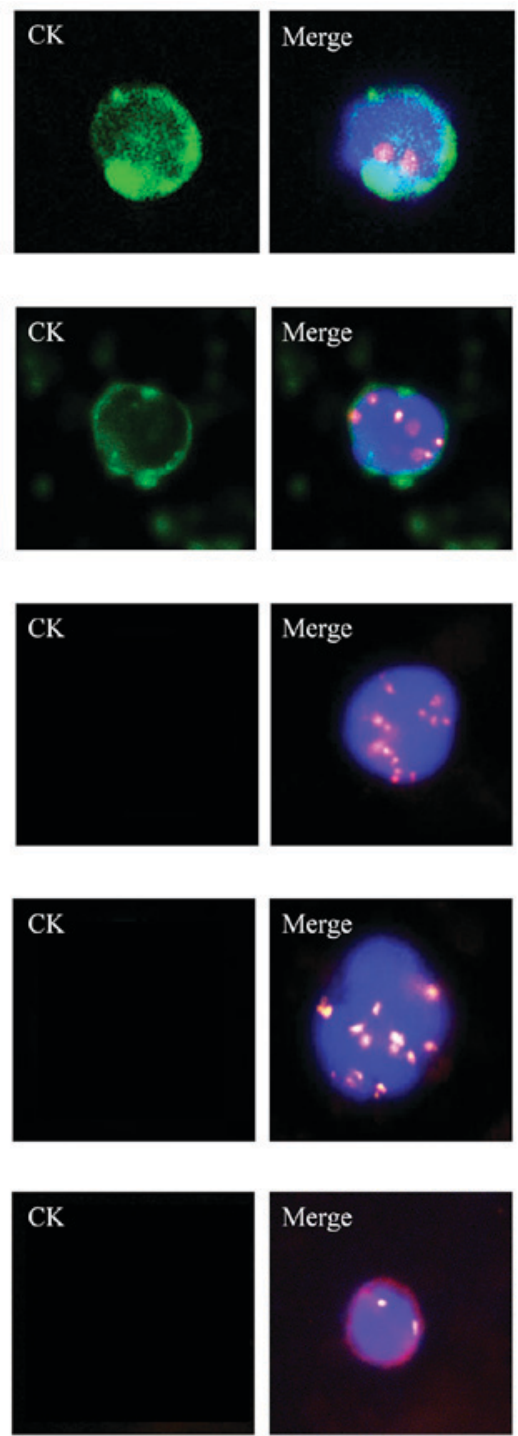

Figure 1. Identification of CTCs in HCC patients using iFISH platform. CK: Green, CEP8: Orange, DAPI: Blue, CD45: Red. (A) CK+/CD45-/DAPI+/CEP8=2; (B) $\mathrm{CK}+/ \mathrm{CD} 45-/ \mathrm{DAPI}+/ \mathrm{CEP} 8>2$; (C) CK-/CD45+/DAPI+/CEP8>2. (D) CTC cluster. (E) CK-/CD45+/DAPI+/CEP8=2, WBC. iFISH, immunostaining-fluorescence in situ hybridization; CTCs, circulating tumor cells; HCC, hepatocellular carcinoma; CK, cytokeratin; CEP8, centromere probe 8; CD, cluster of differentiation; WBC, white blood cell.

Tube containing EDTA and pre-prepared fixative (PBS, 25\% proprietary ingredients, $0.1 \%$ BSA and $0.1 \%$ sodium azide; included in the CellSearch ${ }^{\circledR}$ CTC Kit; Veridex LLC, Raritan, NJ, USA) for protecting CTCs morphology. Subsequently, $6 \mathrm{ml}$ buffer (included in the CellSearch ${ }^{\circledR}$ CTC kit) was added into the tube and centrifuged at room temperature with $164 \mathrm{xg}$ for $10 \mathrm{~min}$, and the supernatant was discarded. The tube was placed in the CellTracks Autoprep System (Menarini Silicon Biosystems, Inc.). EpCAM-coated magnetic beads were used to enrich cells which expressed EpCAM on their surface, and the enriched cells underwent subsequent immunofluorescence staining.

Immunofluorescence reagent (cat no. 7900001; Menarini Silicon Biosystems, Inc.) was used according to the manufacturer's protocol and included anti-CK (CK8, CK18 and CK19; $0.0006 \%$ ) antibody conjugated to phycoerythrin, anti-CD45 antibody $(0.0012 \%)$ conjugated to allophycocyanin and nuclear dye DAPI $(0.005 \%)$. The immunostaining of cells was performed at room temperature for $20 \mathrm{~min}$.

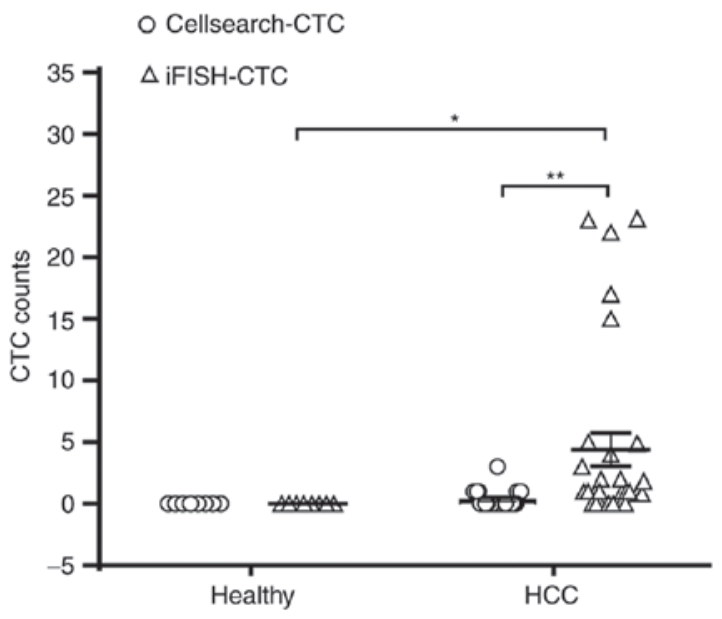

Figure 2. CTCs number detected by Cellsearch ${ }^{\circledR}$ and iFISH ${ }^{\circledR}$ systems in healthy controls and HCC patients. ${ }^{*} \mathrm{P}<0.05$ compared with health controls; ${ }^{* *} \mathrm{P}<0.01$ compared with Ceallsearch ${ }^{\circledR}$. iFISH, immunostaining-fluorescence in situ hybridization; CTCs, circulating tumor cells; HCC, hepatocellular carcinoma. 
Table I. Comparison of discrimination ability between iFISH ${ }^{\circledR}$ and $\mathrm{CellSearch}^{\circledast}$ systems. $^{-}$

\begin{tabular}{lcccccc}
\hline Systems & SEN $(\%)$ & SPE $(\%)$ & NPV & PPV & 95\% CI & P-value \\
\hline Cellsearch-CTCs & 26.67 & 1 & 0.312 & 1 & $0.454-0.813$ & 0.212 \\
iFISH-CTCs & 70 & 1 & 0.526 & 1 & $0.734-0.966$ & $0.001^{\mathrm{a}}$ \\
\hline
\end{tabular}

${ }^{\text {aP }}<0.01$ compared with healthy volunteers. iFISH, immunostaining-fluorescence in situ hybridization; SEN, sensitivity; SPE, specificity; NPV, negative predictive value; PPV, positive predictive value; CI, confidence interval; CTCs, circulating tumor cells.

Finally, cells were transferred to the CellTracks Analyzer II (Cat no. 9555; Menarini Silicon Biosystems, Inc.) for analysis. CTCs were characterized as EpCAM-positive, CK-positive, DAPI-positive and CD45-negative cells (EpCAM+/CK+/DAPI+/CD45-).

Validation of iFISH $^{\circledR}$ platform in cell lines. A cell spiking test was performed to validate the effectiveness of $\mathrm{iFISH}^{\circledR}$ platform in HCC. Huh-7 cells, a hepatoma cell line, was obtained from the cell bank of Shanghai Institutes for Biological Sciences, Chinese Academy of Sciences (Shanghai, China) and cultured in Dulbecco's modified Eagle's medium (Gibco; Thermo Fisher Scientific, Inc., Waltham, MA, USA) in a humidified $5 \% \mathrm{CO}_{2} / 95 \%$ air atmosphere at $37^{\circ} \mathrm{C}$. For the spiking test, 100 Huh-7 cells were added to each $7.5 \mathrm{ml}$ of peripheral blood obtained from the 10 healthy volunteers. Then the aforementioned iFISH ${ }^{\circledR}$ protocol was performed to enrich and count the CTCs detected. The mean recovery rate was calculated by the ratio of iFISH-CTCs number to 100 spiked cells number in the 10 samples.

Statistical analysis. All statistical analyses were performed using SPSS (version 22.0; IBM Corp., Armonk, NY, USA). Data with a normal distribution are presented as the mean \pm the standard deviation, whereas data without a normal distribution are presented as the median (range). Difference between groups was analyzed using the $\chi^{2}$ test, Fisher's exact test or Student's t-test, as appropriate. If variations within groups were not homogeneous, data were analyzed using the nonparametric Mann-Whitney U test or Wilcoxon signed-rank test, as appropriate. The agreement between CellSearch ${ }^{\circledR}$ and iFISH $^{\circledR}$ was determined by $\kappa$ test. Spearman rank correlation analysis was used for nonparametric correlation analysis. Receiver operating characteristic (ROC) analysis was used to analyze the sensitivity (SEN) and specificity of different methods. Positive predictive values and negative predictive values were also computed. Recurrence-free survival (RFS) was defined as the time from LT to the date of conformed recurrence or the final follow-up. Kaplan-Meier survival curves with log-rank test were used to compare the difference in RFS. The threshold of AFP level between high-AFP and low-AFP patients was $400 \mathrm{IU} / \mathrm{ml}$ (4). Univariate Cox proportional hazards regression analysis was performed to identify RFS-associated risk factors. Graphical plots were generated using GraphPad Prism 6.0 (GraphPad Software, La Jolla, CA, USA). $\mathrm{P}<0.05$ was considered to indicate a statistically significant difference.

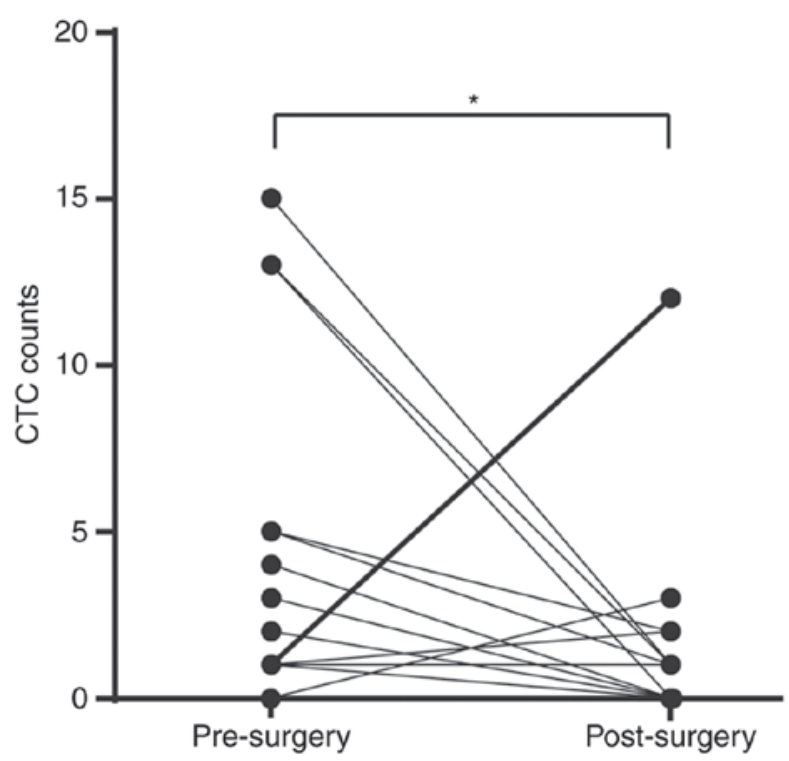

Figure 3. The dynamic changes of iFISH-CTCs counts prior to and 3 months following transplantation in patients with $\mathrm{HCC}$. The thicker line represented the patient who was diagnosed extrahepatic metastasis at the fourth month following LT. "P $<0.05$. iFISH, immunostaining-fluorescence in situ hybridization; CTCs, circulating tumor cells; HCC, hepatocellular carcinoma.

\section{Results}

Technical validation of $i F I S H^{\circledR}$ platform. As iFISH ${ }^{\circledR}$ platform has never been applied in HCC field, to the best of our knowledge, the analytical performance of iFISH for CTCs detection was preliminarily examined in 1 patient and 10 volunteers, as well as cell spiking test. Blood specimens from 10 healthy volunteers were examined and no iFISH-CTCs were detected. Furthermore, one patient with HCC was investigated and exhibited terminal HCC with distant metastases and a number of $7 \mathrm{iFISH-CTCs}$ and CK-/CD45-/DAPI+/CEP8 $>2$ was identified. In the cell spiking test, the mean recovery rate was $81.60 \pm 6.04 \%$, suggesting that iFISH platform was practical for patients with HCC undergoing LT. iFISH-CTCs identified by various characteristics are presented in Fig. 1 .

Detection of CTCs in HCC patients and healthy volunteers. With the exception of single CTCs detected, CTC clusters (defined as CTCs aggregated through plakoglobin-dependent intercellular adhesion) were also detected in certain samples. These CTC clusters are derived from multicellular groupings of primary liver tumor cells. A CTC cluster demonstrated 23 - to 50-fold increased metastatic potential compared 
Table II. Relationship between CTCs positive rate of $\mathrm{iFISH}^{\circledR}$ and Cellsearch ${ }^{\circledR}$ and clinicopathological features of patients with HCC.

\begin{tabular}{|c|c|c|c|c|c|c|c|}
\hline \multirow[b]{2}{*}{ Variable } & \multirow[b]{2}{*}{ Proportion (\%) } & \multicolumn{3}{|c|}{ Cellsearch } & \multicolumn{3}{|c|}{ iFISH } \\
\hline & & Absent & Present & P-value & Absent & Present & P-value \\
\hline \multicolumn{8}{|l|}{ Sex } \\
\hline Male & $27(90)$ & 3 & 0 & \multirow[t]{2}{*}{0.545} & 1 & 2 & \multirow[t]{2}{*}{1} \\
\hline Female & $3(10)$ & 19 & 8 & & 8 & 19 & \\
\hline \multicolumn{8}{|l|}{ Age, years } \\
\hline$\leq 50$ & $12(40)$ & 5 & 5 & \multirow[t]{2}{*}{0.078} & 5 & 5 & \multirow[t]{2}{*}{0.115} \\
\hline$>50$ & $18(60)$ & 17 & 3 & & 4 & 16 & \\
\hline \multicolumn{8}{|l|}{ Etiology } \\
\hline HBV (\%) only & $23(76.67)$ & 17 & 6 & \multirow[t]{3}{*}{0.331} & 7 & 16 & \multirow[t]{3}{*}{0.359} \\
\hline $\mathrm{HCV}(\%)$ only & $3(10)$ & 3 & 0 & & 0 & 3 & \\
\hline Other & $4(13.33)$ & 2 & 2 & & 2 & 2 & \\
\hline \multicolumn{8}{|l|}{ Child-Pugh score } \\
\hline $\mathrm{A} / \mathrm{B}$ & $19(63.33)$ & 13 & 6 & \multirow[t]{2}{*}{0.67} & 6 & 13 & \multirow[t]{2}{*}{1} \\
\hline $\mathrm{C}$ & $11(36.67)$ & 9 & 2 & & 3 & 8 & \\
\hline \multicolumn{8}{|l|}{ AFP, IU/ml } \\
\hline$\leq 400$ & $20(66.67)$ & 13 & 7 & \multirow[t]{2}{*}{0.21} & 2 & 18 & \multirow[t]{2}{*}{$0.002^{\mathrm{a}}$} \\
\hline$>400$ & $10(33.33)$ & 9 & 1 & & 7 & 3 & \\
\hline \multicolumn{8}{|l|}{ Tumor size, cm } \\
\hline$\leq 5$ & $19(63.33)$ & 13 & 2 & \multirow[t]{2}{*}{0.215} & 4 & 11 & \multirow[t]{2}{*}{1} \\
\hline$>5$ & $11(36.67)$ & 9 & 6 & & 5 & 10 & \\
\hline \multicolumn{8}{|l|}{ Tumor number } \\
\hline Single & $18(60)$ & 12 & 6 & \multirow[t]{2}{*}{0.419} & 7 & 11 & \multirow[t]{2}{*}{0.249} \\
\hline Multiple & $12(40)$ & 10 & 2 & & 2 & 10 & \\
\hline \multicolumn{8}{|l|}{ Encapsulation } \\
\hline Complete & $11(36.67)$ & 15 & 5 & 1 & 8 & 12 & 0.204 \\
\hline None & $19(63.33)$ & 7 & 3 & & 1 & 9 & \\
\hline Vascular invasior & & & & & & & \\
\hline No & $23(76.67)$ & 17 & 6 & 1 & 6 & 17 & 0.64 \\
\hline Yes & $7(23.33)$ & 5 & 2 & & 3 & 4 & \\
\hline Portal vein tumo & & & & & & & \\
\hline No & $24(80)$ & 18 & 6 & 0.645 & 7 & 17 & 1 \\
\hline Yes & $6(20)$ & 4 & 2 & & 2 & 4 & \\
\hline BCLC stage & & & & & & & \\
\hline $0+\mathrm{A}$ & $24(80)$ & 13 & 4 & 0.698 & 5 & 12 & 1 \\
\hline $\mathrm{B}+\mathrm{C}$ & $6(20)$ & 9 & 4 & & 4 & 9 & \\
\hline Milan criteria & & & & & & & \\
\hline Within & $10(33.33)$ & 15 & 5 & 1 & 6 & 14 & 1 \\
\hline Beyond & $20(66.67)$ & 7 & 3 & & 3 & 7 & \\
\hline
\end{tabular}

${ }^{\mathrm{a}} \mathrm{P}<0.01$ compared with iFISH-CTCs or Cellsearch-CTCs. iFISH, immunostaining-fluorescence in situ hybridization; CTCs, circulating tumor cells; HCC, hepatocellular carcinoma; AFP, $\alpha$-fetoprotein.

with single CTCs as indicated in previous studies (14). In the present study, it was estimated there would be 1 CTC cluster to every 20 CTCs conservatively. As demonstrated in Fig. 2, the number of CTCs detected in patients with HCC was 0-23/7.5 ml (median, 1/7.5 ml) for iFISH ${ }^{\circledR}$, which was significantly increased compared with that detected by
CellSearch $^{\circledR}(0-2 / 7.5 \mathrm{ml}$, median 0/7.5 ml; P<0.01). CTCs were detected using $\mathrm{iFISH}^{\circledR}$ in $21 / 30$ patients $(70 \%)$, presenting an increased detection rate compared with that in CellSearch ${ }^{\circledR}$ (8/30 patients, positive rate; $26.67 \%)$. The $\kappa$ agreement coefficient between iFISH ${ }^{\circledR}$ and CellSearch ${ }^{\circledR}$ was $0.077(\mathrm{P}=0.559)$, indicating that the similarity in results between Cellsearch 


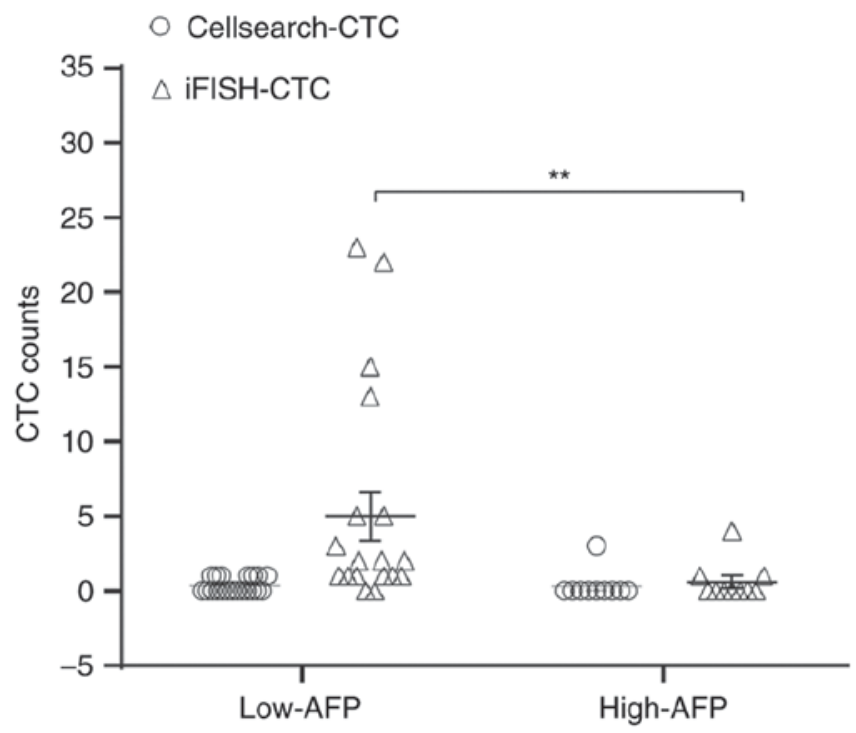

Figure 4. Preoperative CTCs number detected by Cellsearch ${ }^{\circledR}$ and $\mathrm{iFISH}^{\circledR}$ systems in both Low-AFP and High-AFP HCC patients. ${ }^{* *} \mathrm{P}<0.01$. iFISH, immunostaining-fluorescence in situ hybridization; CTCs, circulating tumor cells; HCC, hepatocellular carcinoma; AFP, $\alpha$-fetoprotein.

and iFISH was poor. No CTC was detected in any healthy volunteer for either approach.

To discriminate patents with HCC from healthy volunteers, ROC curves were applied to determine the detection efficiency of iFISH and CellSearch ${ }^{\circledR}$. The SEN of iFISH ${ }^{\circledR}$ was markedly increased compared with that of CellSearch ${ }^{\circledR}$ (SEN, 70 vs. 26.67\%; P<0.01; Table I).

The postoperative levels of CTCs were measured in 23 patients at 3 months following transplantation. Compared with preoperative results, the number of CTCs detected was decreased in 65.21 and $34.78 \%$ (iFISH $^{\circledR}$ and CellSearch ${ }^{\circledR}$, respectively) of patients with $\mathrm{HCC}$ following LT. However, the iFISH-CTCs level didn't change in $21.73 \%$ of patients and increased in $13.04 \%$. For Cellsearch-CTCs, the ratio is 56.52 and $8.70 \%$ respectively (Fig. 3). iFISH-CTCs count was significantly decreased following transplantation $(\mathrm{P}<0.05)$. Notably, the patient whose iFISH-CTCs counts increased from $2 / 7.5 \mathrm{ml}$ preoperatively to $12 / 7.5 \mathrm{ml}$ postoperatively was diagnosed with extrahepatic metastasis 4 months following surgery. There was no similar elevation detected in the Cellsearch ${ }^{\circledR}$-CTCs count.

Associations between positive rate of CTCs and clinicopathological characteristics. The associations between positive rates of both preoperative Cellsearch-CTCs and iFISH-CTCs and clinicopathological characteristics of HCC patients were examined.

Results identified a significant negative association between iFISH-CTCs positive rate and high-AFP rate (Spearmans correlation value $=-0.617 ; \mathrm{P}<0.001)$. However, no significant associations were observed between CTCs and other clinicopathological features including AFP level, vascular invasion, portal vein tumor thrombus, BCLC stage and Milan criteria (Table II).

Notably, results demonstrated that the median preoperative iFISH-CTCs number in low-AFP and high-AFP patients was $1.5 / 7.5 \mathrm{ml}$ (range, 0-23) and 0/7.5 $\mathrm{ml}$ (range, 0-4), respectively, which suggested that the low-AFP subgroup had a significantly increased iFISH-CTCs level compared with the high-AFP group ( $\mathrm{P}<0.01$, Fig. 4). Noticeably, positive iFISH-CTCs were detected in $90 \%$ of patients with low-AFP levels. Spearman correlation analysis demonstrated that AFP levels were negatively associated with iFISH-CTCs levels $(\mathrm{R}=-0.385 ; \mathrm{P}<0.05)$.

Prognostic value of CTCs in HCC patients. At a medium follow-up of 15 months (range 4-20), 11 patients with HCC $(36.67 \%)$ were diagnosed with intrahepatic recurrence $(n=7)$ or extrahepatic metastasis using CT or MRI scanning. Furthermore, metastatic lesions were located in numerous locations including the bone $(n=1)$, lung $(n=1)$, abdominal cavity $(n=1)$ and the adrenal gland $(n=1)$, which were detected by CT, MRI or bone scanning. Univariate Cox proportional hazards regression analysis identified that preoperative iFISH-CTCs $(\geq 5 / 7.5 \mathrm{ml})$ and vascular invasion were prognostic factors for RFS (Table III). The median RFS in patients with iFISH-CTCs $<5 / 7.5 \mathrm{ml}$ was significantly increased compared with CTC $\geq 5 / 7.5 \mathrm{ml}$ (15 vs. 5.5 months). Kaplan-Meier analysis demonstrated that preoperative iFISH-CTCs $\geq 5 / 7.5 \mathrm{ml}$ was associated with poor RFS (hazard ratio=5.142, $\mathrm{P}<0.01$; Table III, Fig. 5A). For patients with low-AFP (AFP<400 IU/ml) levels, the prognostic value was increased (hazard ratio $=26.4, \mathrm{P}<0.001$; Fig. 5B) comparing with high-AFP patients.

\section{Discussion}

In the present study, the analytical performance and clinical value of iFISH $^{\circledR}$ in patients with HCC undergoing LT was evaluated. The detected iFISH-CTCs count was markedly increased compared with that of Cellsearch-CTCs, and the positive rate of iFISH-CTCs was significantly increased compared with Cellsearch-CTCs. Furthermore, the positive rate of Cellsearch-CTCs in the present study was significantly decreased compared with previous studies investigating patients with HCC who underwent hepatectomy (15-17). One possible reason for this is that the tumor lesion was drawn out thoroughly during LT; however, following hepatectomy, lesions may remain in residual liver lobes. Thus, for patients with HCC undergoing LT, the increased detection sensitivity may facilitate in the clinical application of the iFISH $^{\circledR}$ platform.

The ability of $\mathrm{iFISH}^{\circledR}$ system to discriminate between HCC patients and healthy volunteers may provide a promising method for diagnosing and/or monitoring HCC. Currently, AFP remains the mainstream serum screening biomarker for HCC. However, $30-40 \%$ of patients with HCC are AFP-negative $(18,19)$, and it has been previously demonstrated that pregnancy, benign liver disease and certain gastrointestinal tumors may contribute to false-positive result $(4,20)$. Therefore, guidelines in the USA and Europe have recommended ultrasonography alone without AFP examination as the routine surveillance method for $\operatorname{HCC}(21,22)$. Notably, in the present study results demonstrated that there was an increased negative association between iFISH-CTCs positive rate and high-AFP rate. This association was evident as one or more iFISH-CTCs were detected in $90 \%$ of low-AFP patients. 
Table III. Univariate analysis of factors predictive for RFS. Preoperative iFISH-CTCs $\geq 5 / 7.5$ ml and vascular invasion were prognostic factors for RFS.

\begin{tabular}{lccc}
\hline Factors & Hazard ratio & 95\% CI & P-value \\
\hline Univariate analysis & & & $0.629-6.824$ \\
AFP, IU/ml (<400 vs. >400) & 2.072 & $0.115-2.486$ & 0.231 \\
Cellsearch-CTC (negative vs. positive) & 0.536 & $1.528-17.305$ & 0.425 \\
iFISH-CTC (<5 vs. >5) & 5.142 & $0.78-47.877$ & $0.008^{\mathrm{b}}$ \\
Milan criteria (within vs. beyond) & 6.11 & $0.857-10.373$ & 0.085 \\
Portal vein tumor thrombus (negative vs. positive) & 2.982 & $0.559-7.959$ & 0.086 \\
Tumor number (single vs. multiple) & 2.109 & $1.290-14.467$ & 0.271 \\
Vascular invasion (negative vs. positive) & 4.32 & $0.018^{\mathrm{a}}$
\end{tabular}

${ }^{\mathrm{a}} \mathrm{P}<0.05$ compared with iFISH-CTCs; ${ }^{\mathrm{P}}<0.01$ compared with iFISH-CTCs. iFISH, immunostaining-fluorescence in situ hybridization; CTCs, circulating tumor cells; RFS, recurrence-free survival; ATP, $\alpha$-fetoprotein.
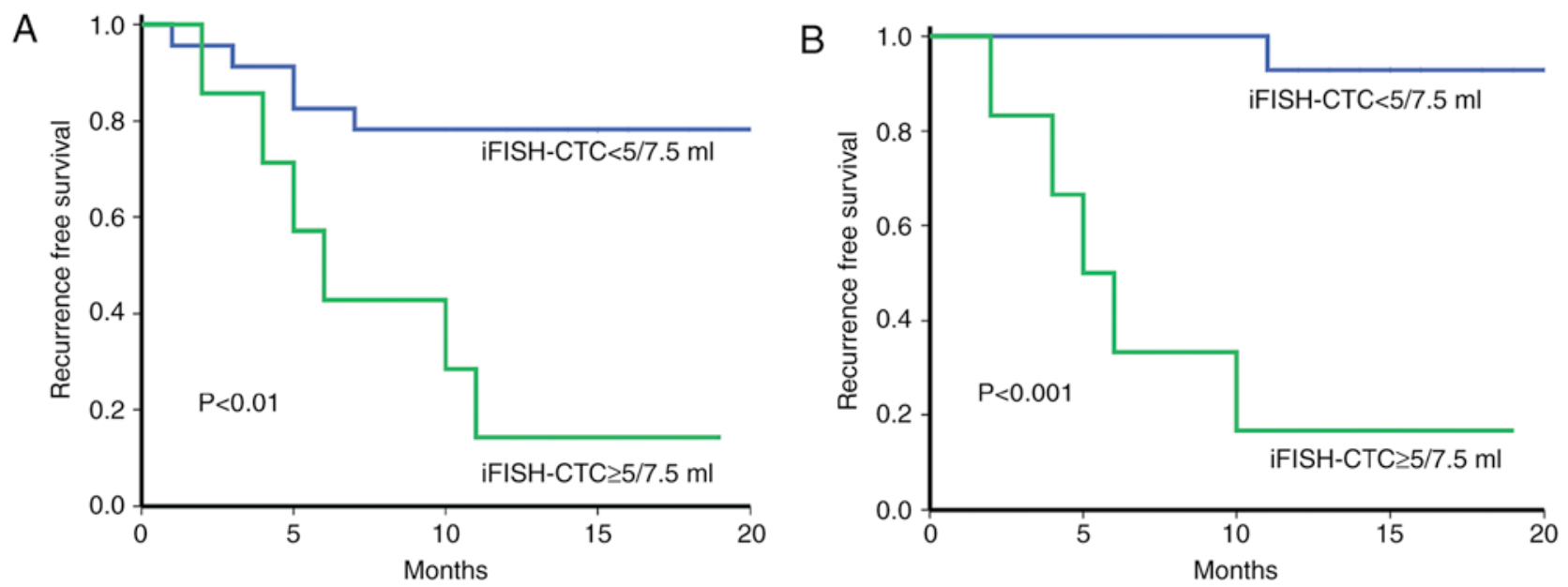

Figure 5. Kaplan-Meier analysis of RFS in patients with different level of iFISH-CTCs and AFP. (A) Patients with lower preoperative iFISH-CTC count $(<5 / 7.5 \mathrm{ml})$ had increased RFS compared with those with iFISH-CTC $\geq 5 / 7.5 \mathrm{ml}(\mathrm{P}<0.01)$. (B) Patients with AFP $<400 \mathrm{IU} / \mathrm{ml}$, patients with preoperative iFISH-CTC count $<5 / 7.5 \mathrm{ml}$ had significantly increased RFS compared with those with iFISH-CTC $\geq 5 / 7.5 \mathrm{ml}$ ( $\mathrm{P}<0.001$ ). RFS, recurrence-free survival; iFISH, immunostaining-fluorescence in situ hybridization; CTCs, circulating tumor cells; HCC, hepatocellular carcinoma; AFP, $\alpha$-fetoprotein.

Thus, iFISH-CTCs levels may provide an effective supplement for the AFP approach, especially for those patients with low-AFP levels.

The present study also examined the dynamic change of CTCs during the perioperative period. Surgical manipulation including squeezing or traction on the tumor has been proven to facilitate tumor cell dissemination into circulation (23). To the best of our knowledge, this is the first time that a significant decrease of iFISH-CTCs load was observed following LT, which may be caused by the radical removal of the primary tumor. However, future studies should be implemented to investigate the value of monitoring CTCs changes along with postoperative treatment. According to the present study, the regular surveillance of iFISH-CTCs following LT counts may be effective for early-detection of HCC recurrence, and subsequently inform personalized antitumor therapy.

The prognostic value of iFISH $^{\circledR}$ platform was also investigated. Results of the present study demonstrated that iFISH $^{\circledR}$-CTCs $(\geq 5 / 7.5 \mathrm{ml})$ was a prognostic factor for patients with HCC undergoing LT. Despite this, Cellsearch ${ }^{\circledR}$-CTCs have been previously reported as a strong predictor for HCC recurrence following curative resection (15-17); however, the prognostic value of Cellsearch ${ }^{\circledR}-\mathrm{CTC}$ s was not favorable in the present study, which may be due to the low detection rate. Additionally, it was also identified that in addition to vascular invasion, portal vein tumor thrombus, BCLC stage and Milan criteria, iFISH-CTCs is an independent prognostic predictor for patients with HCC undergoing LT. Notably, Milan criteria has been previously reported as a well-known predictor for HCC recurrence following transplantation (13). However, in the present study, results demonstrated that only $50 \%$ $(10 / 20)$ of patients exceeding Milan criteria suffered relapse. Considering the lack of serum biomarker in Milan Criteria, iFISH-CTCs count may be a promising option.

To conclude, iFISH ${ }^{\circledR}$ platform presents an increased analytical sensitivity compared with the Cellsearch ${ }^{\circledR}$ platform and has the potential to be a dynamic monitoring tool for CTCs, whose level in peripheral circulation may be a prognostic marker for patients with HCC undergoing LT. 


\section{Competing interests}

The authors declare that they have no competing interests.

\section{References}

1. Jemal A, Bray F, Center MM, Ferlay J, Ward E and Forman D: Global cancer statistics. CA Cancer J Clin 61: 69-90, 2011.

2. Trojan J, Zangos S and Schnitzbauer AA: Diagnostics and treatment of hepatocellular carcinoma in 2016: Standards and developments. Visc Med 32: 116-120, 2016.

3. Welker MW, Bechstein WO, Zeuzem S and Trojan J: Recurrent hepatocellular carcinoma after liver transplantation-an emerging clinical challenge. Transpl Int 26: 109-118, 2013.

4. Rich N and Singal AG: Hepatocellular carcinoma tumour markers: Current role and expectations. Best Pract Res Clin Gastroenterol 28: 843-853, 2014.

5. Gorges TM, Tinhofer I, Drosch M, Röse L, Zollner TM, Krahn T and von Ahsen O: Circulating tumour cells escape from EpCAM-based detection due to epithelial-to-mesenchymal transition. BMC Cancer 12: 178, 2012.

6. Schulze K, Gasch C, Staufer K, Nashan B, Lohse AW, Pantel K, Riethdorf S and Wege H: Presence of EpCAM-positive circulating tumor cells as biomarker for systemic disease strongly correlates to survival in patients with hepatocellular carcinoma. Int J Cancer 133: 2165-2171, 2013.

7. Sheng Y, Wang T, Li H, Zhang Z, Chen J, He C, Li Y, Lv Y, Zhang J, Xu C, et al: Comparison of analytic performances of Cellsearch and iFISH approach in detecting circulating tumor cells. Oncotarget 8: 8801-8806, 2017.

8. Jiang J, Wang DD, Yang M, Chen D, Pang L, Guo S, Cai J, Wery JP, Li L, Li HQ and Lin PP: Comprehensive characterization of chemotherapeutic efficacy on metastases in the established gastric neuroendocrine cancer patient derived xenograft model. Oncotarget 6: 15639-15651, 2015.

9. Ma C, Lv Y, Jiang R, Li J, Wang B and Sun L: Novel method for the detection and quantification of malignant cells in the CSF of patients with leptomeningeal metastasis of lung cancer. Oncol Lett 11: 619-623, 2016.

10. Gao Y, Zhu Y, Zhang Z, Zhang C, Huang X and Yuan Z: Clinical significance of pancreatic circulating tumor cells using combined negative enrichment and immunostaining-fluorescence in situ hybridization. J Exp Clin Cancer Res 35: 66, 2016.

11. Lin PP: Integrated EpCAM-independent subtraction enrichment and iFISH strategies to detect and classify disseminated and circulating tumors cells. Clin Transl Med 4: 38, 2015.

12. Llovet JM, Bru C and Bruix J: Prognosis of hepatocellular carcinoma: The BCLC staging classification. Semin Liver Dis 19: $329-338,1999$
13. Mazzaferro V, Regalia E, Doci R, Andreola S, Pulvirenti A, Bozzetti F, Montalto F, Ammatuna M, Morabito A and Gennari L: Liver transplantation for the treatment of small hepatocellular carcinomas in patients with cirrhosis. N Engl J Med 334: 693-699, 1996.

14. Aceto N, Bardia A, Miyamoto DT, Donaldson MC, Wittner BS, Spencer JA, Yu M, Pely A, Engstrom A, Zhu H, et al: Circulating tumor cell clusters are oligoclonal precursors of breast cancer metastasis. Cell 158: 1110-1122, 2014.

15. Felden J, Schulze K, Krech T, Ewald F, Nashan B, Pantel K, Lohse AW, Riethdorf S and Wege H: Circulating tumor cells as liquid biomarker for high HCC recurrence risk after curative liver resection. Oncotarget 8: 89978-89987, 2017.

16. Guo W, Yang XR, Sun YF, Shen MN, Ma XL, Wu J, Zhang CY, Zhou Y, Xu Y, Hu B, et al: Clinical significance of EpCAM mRNA-positive circulating tumor cells in hepatocellular carcinoma by an optimized negative enrichment and qRT-PCR-based platform. Clin Cancer Res 20: 4794-4805, 2014.

17. Sun YF Xu Y, Yang XR, Guo W, Zhang X, Qiu SJ, Shi RY, Hu B, Zhou J and Fan J: Circulating stem cell-like epithelial cell adhesion molecule-positive tumor cells indicate poor prognosis of hepatocellular carcinoma after curative resection. Hepatology 57: 1458-1468, 2013

18. Trevisani F, D'intino PE, Morselli-Labate AM, Mazzella G, Accogli E, Caraceni P, Domenicali M, De Notariis S, Roda E and Bernardi M: Serum alpha-fetoprotein for diagnosis of hepatocellular carcinoma in patients with chronic liver disease: Influence of HBsAg and anti-HCV status. J Hepatol 34: 570-575, 2001.

19. Debruyne EN and Delanghe JR: Diagnosing and monitoring hepatocellular carcinoma with alpha-fetoprotein: New aspects and applications. Clin Chim Acta 395: 19-26, 2008.

20. Abdul-Hamid S, Fox R and Martin I: Maternal serum screening for trisomy 21 in women with a false positive result in last pregnancy. J Obstet Gynaecol 24: 374-376, 2004.

21. Bruix J and Sherman M; American Association for the Study of Liver Diseases: Management of hepatocellular carcinoma: An update. Hepatology 53: 1020-1022, 2011.

22. European Association for the Study of The Liver; European Organisation For Research and Treatment of Cancer: EASL-EORTC clinical practice guidelines: Management of hepatocellular carcinoma. J Hepatol 56: 908-943, 2012.

23. Zhang Y, Shi ZL, Yang X and Yin ZF: Targeting of circulating hepatocellular carcinoma cells to prevent postoperative recurrence and metastasis. World J Gastroenterol 20: 142-147, 2014.

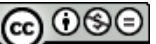

This work is licensed under a Creative Commons Attribution-NonCommercial-NoDerivatives 4.0 International (CC BY-NC-ND 4.0) License. 